\title{
A model for torque losses in variable displacement axial piston motors
}

\author{
G-A. Moslått ${ }^{1}$ M.R. Hansen ${ }^{2}$ N.S. Karlsen ${ }^{3}$ \\ ${ }^{1}$ University of Agder and National Oilwell Varco, 4898 Grimstad, Norway. \\ E-mail: geir.a.moslatt@uia.no \\ ${ }^{2}$ Department of Engineering, University of Agder, 4879 Grimstad, Norway. \\ E-mail: michael.r.hansen@uia.no \\ ${ }^{3}$ Bosch Rexroth AS, 1405 Langhus, Norway. \\ E-mail: Nicolai.Karlsen@boschrexroth.no
}

\begin{abstract}
This paper includes a comparison of earlier presented models for torque losses in hydraulic motors and several proposed models that all rely on data typically available for a system engineer. The new models and the old ones are compared. The new models are all based on a model developed by Jeong Jeong (2007);Jeong and Kim (2007) with an expansion that include variable displacement. All of the new models yield very good accuracy down to approximately $50 \%$ of maximum displacement and down to approximately $15 \%$ of maximum speed. In these operational ranges the deviation in torque is less than $1 \%$. The main purpose of the new models is to facilitate simulations of hydraulically actuated winches with a balance between accuracy and model complexity. This purpose is considered fulfilled with several of the proposed models.
\end{abstract}

Keywords: Hydraulics, torque loss, axial piston motor, winch drive, offshore knuckle boom cranes, Bosch Rexroth.

\section{Introduction}

The use of mathematical models for hydraulic motors are useful in the industry to estimate losses in a design phase and to be included in simulation models. The main challenge with the use of previously generated research and models in this field is the complexity of the models, especially models that take into account variable displacement. The complexity makes the models harder to use and may increase the cost of computations heavily. This is a huge disadvantage in a design phase when several system configurations, load cases and control algorithms must be evaluated.

In the offshore business today there is a high demand for hydraulic drives in crane and drilling applications. This is especially relevant for large knuckle boom cranes with safe working loads ranging from 80 to 900 metric tons. At the same time the manufacturers have the need to stay competitive both in performance and cost. To maintain a good position in the market the manufacturer mainly has three challenges.

1: Minimization of equipment costs. A system with optimal choice and sizing of components is needed to keep the costs down, however, each delivery is typically tailor-made and there are no prototypes available for testing. This automatically puts more emphasis on modeling and simulation in the design phase.

2: Behavior prediction. It is important for the manufacturers, but also a need from customers (platform operators) to have simulation models that can predict the performance of the equipment under different weather 
conditions that are not easily reproduced in tests. Such models can be used in the design phase, virtual testing, and lift preparations. An example is the increasing use of models to engineer subsea lifts for large offshore cranes. These models need a certain accuracy to get approval from third party certification authorities.

3: Minimize test costs. It is a challenge to keep the test time at a minimum especially because there is a trend that crane features are constantly increasing in number and complexity. The manufacturer needs the test time to be as short and effective as possible. Test time is one of the factors that has an important impact on the final cost of a project. Testing is mainly divided into two categories; inhouse testing and real life testing. The first one is done at an office facility and is orders of magnitude less costly than the second one. Due to this, the inhouse testing is preferred when possible. A common factor in all these challenges is that they can all be handled much better with increased component and system knowledge that is implemented in time domain simulation models.

When predicting behavior of a crane it is important to understand what kind of losses and loss properties the different hydraulic actuators have. The focus of this paper is the hydraulic motors of the main winch system found in all offshore knuckle boom cranes. A typical knuckle boom crane design is seen in fig. 1 .

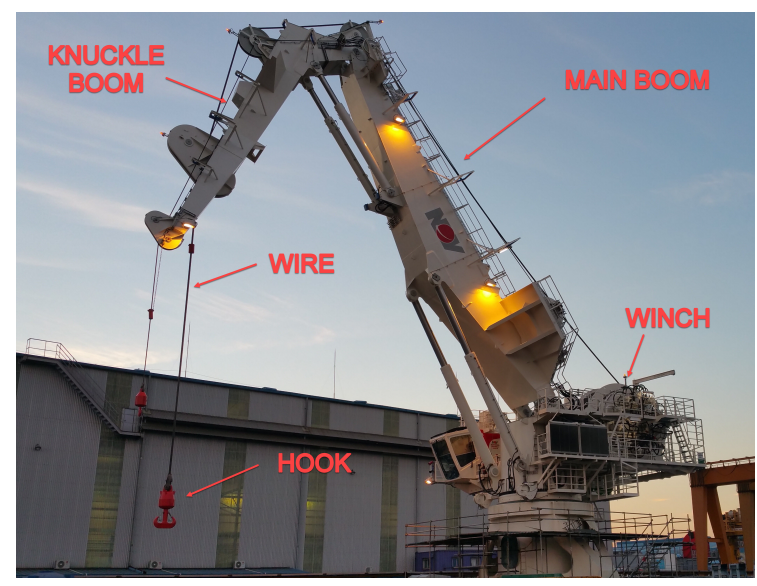

Figure 1: Typical knuckle boom crane design.

There is an increasing demand for accurate modeling of these winch systems to predict the performance during heave compensation, during tension control and when moving payloads through the splash zone. To build these types of models it is important to be able to predict the nonlinear behavior associated with friction and leakage, also referred to as hydromechanical and volumetric losses in hydraulics. The focus of this article is the friction losses in the variable displacement axial piston hydraulic motors of the winch systems, see fig 2 .

The overall friction losses are complex combinations of turbulent friction, viscous friction, mixed lubrication friction and dry friction. Despite the importance of accurate modeling there is no unique model of the overall friction loss of hydraulic piston motors with variable displacement. This paper presents a modeling technique that is useful from a crane manufacturers point of view. This means that the following criteria are considered:

- Reasonable trade-off between accuracy and number of parameters

- Computational efficient without introducing complex sub-models concerned with tribology, thermodynamics and structural deformations.

- Developed in cooperation with sub-supplier (motor manufacturer).

- Motor specific.

The paper will investigate the models presented in literature and new models presented in this paper with a view to identify a model that meets the above criteria in the best possible way.

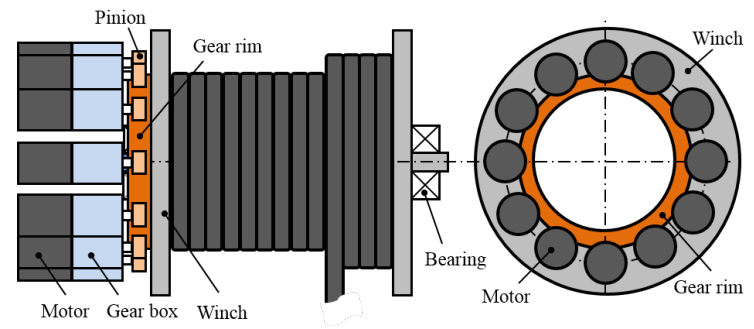

Figure 2: Simple sketch of a winch system.

\section{State of the art}

The total friction in winch systems has not attracted any research effort, however, the hydraulic motors is a topic subjected to numerous studies. Among the first researchers in this field were Wilson Wilson (1948) and Schlösser Schlösser (1961). Both made some basic and simple models and, normally, both models fail to yield a decent accuracy for the complete 3-dimensional work space of a motor comprising speed, torque and displacement ranges. Due to the lack of accuracy, several methods for model generation have been studied. They can be divided into three categories;

- models based on physical interactions in pump/motor 
- numerical models based on curve fitting to experimental data

- analytic models that are based on both numerical and physical models

Huhtala made a comparison in 1997 Huhtala and Villenius (1997) of his own numerical (the two line model) approach with Wilsons physical and Doreys Dorey (1988) analytical approach. He showed that the existing fixed parameter models from Wilson and Dorey did not yield a satisfactory accuracy. Results were especially poor across the displacement range. Huhtala on the other hand, used a numerical approach based on measurements that gave a significantly better accuracy but also introduced 14 parameters for fixed displacement. Ortwig Ortwig (2002) and JeongJeong and Kim (2007) did some work establishing an overview over the different losses in the motor. Based on this knowledge, they made expressions based on assumptions on geometry and tolerances in the motor. Of these advanced expressions Ortwig chose to simplify with a numerical representation of the total loss, while Jeong kept the physical terms and combined them.

\section{Model Development}

In this section the most common friction loss models are briefly discussed and a number of new models are introduced. The models are presented in the following sections 3.1 to 3.13. The friction torque, $T_{f}$, is the absolute friction torque working in opposite direction of $\omega_{m}$. Hence it can be assumed that all terms in the equations presented can be multiplied with $\operatorname{sign}\left(\omega_{m}\right)$.

\subsection{Wilson}

In 1948 Wilson Wilson (1948) presented a relatively simple model for friction losses in constant displacement motors. The model is grouped into three parts; dry friction proportional with shaft torque, viscous friction proportional with viscosity and speed, and a constant torque loss.

$$
\begin{array}{r}
T_{f}=C_{a} \cdot D_{m, \max } \cdot \Delta p_{m}+C_{b} \cdot \mu \cdot D_{m, \max } \cdot\left|\omega_{m}\right| \\
+C_{c} \cdot D_{m, \max }
\end{array}
$$

\subsection{Schlösser}

Schlösser Schlösser (1961) replaced Wilsons last term with a term for acceleration of the liquid. He assumed a loss term proportional with the square of the tangential velocity of the revolving barrel.

$$
\begin{aligned}
T_{f}=C_{a} \cdot D_{m, \max } \cdot \Delta p_{m}+ & C_{b} \cdot \mu \cdot D_{m, \max } \cdot\left|\omega_{m}\right| \\
& +C_{d} \cdot D_{m, \text { max }}^{5 / 3} \cdot \rho \cdot \omega^{2}
\end{aligned}
$$

\subsection{Thoma}

In 1969 Jean Thoma Thoma (1969) made a modification of the Schlösser model by including a displacement variable, $\alpha_{m}$, for the liquid acceleration losses. Although the displacement dependency is included for the liquid acceleration losses, the displacement setting is still neglected with regards to the viscous losses in the second term.

$$
\begin{array}{r}
T_{f}=C_{a} \cdot D_{m, \max } \cdot \Delta p_{m}+C_{b} \cdot \mu \cdot D_{m, \max } \cdot\left|\omega_{m}\right| \\
+C_{e} \cdot \alpha_{m}^{3} \cdot D_{m, \max }^{5 / 3} \cdot \rho \cdot \omega^{2}
\end{array}
$$

\subsection{Pacey}

In 1979 Pacey Pacey et al. (1979) presented a modification of the Wilson model to include different displacement settings. He basically took the Wilson model and added $\frac{1-\tan \alpha_{m, \max }}{1-\tan \alpha_{m}}$, in the dry and viscous friction terms.

$$
\begin{aligned}
& T_{f}=C_{a} \cdot D_{m, \max } \cdot \Delta p_{m} \cdot \frac{1-\tan \left(\alpha_{m, \max }\right)}{1-\tan \left(\alpha_{m}\right)} \\
& +C_{b} \cdot \mu \cdot\left|\omega_{m}\right| \cdot D_{m} \cdot \frac{1-\tan \left(\alpha_{m, \max }\right)}{1-\tan \left(\alpha_{m}\right)}+C_{e}
\end{aligned}
$$

\subsection{Huhtala}

In 1997 Huhtala Huhtala and Villenius (1997) did a review of the torque loss models of Wilson, Schlösser, Thoma, Zarotti, Dorey, and Rydberg. Huhtala saw the need for better accuracy and a model that would represent the whole working range with regards of pressure, speed and displacement. The model he introduced was based on multiple polynomial fitting and measurements. He reported a better accuracy than Dorey and Thoma, but increased the amount of parameters significantly. For fixed motor displacement you need 14 parameters distributed over four different curve fitted polynomials. The concept is according to Huhtala prepared to represent variable displacement motors, but details are not given and how to adapt is unclear to authors of this paper. Regardless of how to implement, the complexity will increase and several additional curve fitted polynomials will be needed.

\subsection{Ortwig}

Ortwig Ortwig (2002) did a study in where he investigated 13 different loss terms for describing the total loss in a hydraulic motor. The terms should cover laminar and turbulent flow losses, pulse losses, churning, losses, friction losses at valve plate, piston, roller bearings, seals, mixed bearing friction and solid friction. 
Based on these formulas he formed a torque interpolation equation (eq. 5) in exchange for the set of formulas that described each loss term separately. He proved that his new equation gave similar accuracy.

$$
\begin{aligned}
& T_{f}=C_{e}+C_{f} \cdot \Delta p_{m}+C_{g} \cdot\left|\omega_{m}\right|+C_{h} \cdot\left|\omega_{m}\right| \cdot \Delta p_{m} \\
& +C_{i} \cdot \omega_{m}^{2}+C_{j} \cdot \alpha_{m}+C_{k} \cdot \alpha_{m} \cdot \Delta p_{m}+C_{l} \cdot \alpha \cdot\left|\omega_{m}\right|
\end{aligned}
$$

\subsection{Jeong}

A physical model for torque loss was introduced in 2007 by Jeong Jeong and Kim (2007)Jeong (2007). The model considers terms for friction due to forces in moving gaps (piston vs piston block, swash-plate vs slipper, piston block vs port-plate), turbulent port plate inlet loss, port plate notch losses (also assumed turbulent), churning losses and bearing losses. There are similarities between the terms in Jeong and Ortwigs model derivation, but Ortwig ended up simplifying to a numerical model. Jeong simplified his expression, but kept the physical meaning by combining terms with similar parameter dependencies. Unlike Ortwig, Jeong only considered a fixed displacement motor.

$$
\begin{array}{r}
T_{f}=C_{e}+C_{f} \cdot \Delta p_{m}+C_{g} \cdot \mu \cdot\left|\omega_{m}\right|+C_{i} \cdot \rho \cdot \omega_{m}^{2} \\
+C_{k} \cdot \frac{\Delta p_{m}}{\left|\omega_{m}\right|} \cdot \sqrt{\frac{\Delta p_{m}}{\rho}}+C_{m} \cdot \Delta p_{m}{ }^{2}
\end{array}
$$

In this case the $C_{e}$ term represents Coulomb friction in motor and motor bearings. The $C_{f}$ term includes pressure dependent bearing losses and piston friction. The $C_{g}$ term represents viscous losses in bearings, churning losses for the cylinder block, viscous slipper friction and viscous friction between barrel and port plate. Port plate inlet loss and churning losses from pistons and slippers are presented in term $C_{i} . C_{k}$ represent the losses due to valve notches in the port plate and $C_{m}$ is to account for mixed lubrication friction at high pressures.

\subsection{Modified Jeong 1 (MJ1)}

When forming new alternatives it was chosen to use Jeongs model as a base. Jeongs model was first just modified slightly to account for variable displacement. The effect of these changes are that the term $C_{i}$ no longer represents both port plate inlet loss and churning losses in a realistic sense, since churning loss is independent of displacement setting.

$$
\begin{aligned}
T_{f}=C_{e} & +C_{f} \cdot \Delta p_{m}+C_{g} \cdot \mu \cdot\left|\omega_{m}\right|+C_{m} \cdot \Delta p_{m}{ }^{2} \\
& +C_{i} \cdot \rho \cdot \omega_{m}^{2} \cdot D_{m}^{3}+C_{k} \cdot \frac{\Delta p_{m}}{\left|\omega_{m}\right|} \cdot \sqrt{\frac{\Delta p_{m}}{\rho}}
\end{aligned}
$$

\subsection{MJ2}

Next alternative is made to evaluate the impact of removing the losses caused by the turbulent flow through the port notches $\left(C_{k}\right)$.

$$
\begin{aligned}
T_{f}= & C_{e}+C_{f} \cdot \Delta p_{m}+C_{g} \cdot \mu \cdot\left|\omega_{m}\right| \\
& +C_{i} \cdot \rho \cdot \omega_{m}^{2} \cdot D_{m}^{3}+C_{m} \cdot \Delta p_{m}{ }^{2}
\end{aligned}
$$

\section{$3.10 \mathrm{MJ3}$}

For MJ3 we removed the term for high pressure boundary and mixed friction $\left(C_{m}\right)$.

$$
\begin{array}{r}
T_{f}=C_{e}+C_{f} \cdot \Delta p_{m}+C_{g} \cdot \mu \cdot\left|\omega_{m}\right| \\
+C_{i} \cdot \rho \cdot \omega_{m}^{2} \cdot D_{m}^{3}
\end{array}
$$

\section{$3.11 \mathrm{MJ4}$}

At this point the $C_{f}$ term is changed such that it varies with displacement. In terms of the physical nature of the $C_{f}$-term, it does no longer directly represent the pressure dependent losses in pistons and bearings.

$$
\begin{aligned}
T_{f}=C_{e} & +C_{f} \cdot \Delta p_{m} \cdot D_{m}+C_{g} \cdot \mu \cdot\left|\omega_{m}\right| \\
& +C_{i} \cdot \rho \cdot \omega_{m}^{2} \cdot D_{m}^{3}+C_{m} \cdot \Delta p_{m}{ }^{2}
\end{aligned}
$$

\section{$3.12 \mathrm{MJ5}$}

With alternative 3 (eq.9) as a base, alternative 5 has the exponential in term $C_{i}$ as a variable parameter. The term is representing the turbulent inlet flow into the chamber. With a perfect turbulent inlet flow the $C_{o}$ factor will be equal 2 . The opposite would be an exponential set to 1 which is used for laminar flow.

$$
\begin{array}{r}
T_{f}=C_{e}+C_{f} \cdot \Delta p_{m} \cdot D_{m}+C_{g} \cdot \mu \cdot\left|\omega_{m}\right| \\
+C_{i} \cdot \rho \cdot\left|\omega_{m}\right|^{C_{n}} \cdot D_{m}^{C_{n}+1}+C_{m} \cdot \Delta p_{m}{ }^{2}
\end{array}
$$

\section{$3.13 \mathrm{MJ6}$}

As an alternative 6 the notch flow loss is set back on but now also dependent on displacement.

$$
\begin{aligned}
T_{f}= & C_{e}+C_{f} \cdot \Delta p_{m} \cdot D_{m}+C_{g} \cdot \mu \cdot\left|\omega_{m}\right|+C_{m} \cdot \Delta p_{m}{ }^{2} \\
& +C_{i} \cdot \rho \cdot \omega_{m}^{2} \cdot D_{m}^{3}+C_{k} \cdot \frac{D_{m} \cdot \Delta p_{m}}{D_{m, \max } \cdot\left|\omega_{m}\right|} \cdot \sqrt{\frac{\Delta p_{m}}{\rho}}
\end{aligned}
$$




\subsection{Comparison}

To compare a selection of different models, the model parameters were all determined based on efficiency data from Bosch Rexroth Bosch Rexroth AG (2010) using numerical minimization of the deviation between modeling results and experimental data. A model comparison with the a A6VMs71 280 motor was done first with a fixed displacement setting at $280 \mathrm{~cm}^{3} / \mathrm{rev}$, and then for a whole range of displacement settings. Oil density and viscosity can be considered constants.

Table 1: Basic info, friction models

\begin{tabular}{lcccc}
\hline Author & Year & Model Type & Motor type & Par. \\
\hline Wilson & 1948 & Physical & Fixed & 3 \\
Sclosser & 1961 & Physical & Fixed & 3 \\
Thoma & 1969 & Physical & Variable & 3 \\
Pacey & 1979 & Physical & Variable & 3 \\
Huhtala & 1997 & Numerical & Variable & 28 \\
Ortwig & 2002 & Numerical & Variable & 8 \\
Jeong & 2007 & Physical & Fixed & 6 \\
MJ1 & 2017 & Physical & Variable & 6 \\
MJ2 & 2017 & Physical & Variable & 5 \\
MJ3 & 2017 & Physical & Variable & 4 \\
MJ4 & 2017 & Phys- and numerical & Variable & 5 \\
MJ5 & 2017 & Phys- and numerical & Variable & 6 \\
MJ6 & 2017 & Phys- and numerical & Variable & 5 \\
\hline
\end{tabular}

For the first comparison (fig 3), parameter identification is performed for a fixed displacement setting of A6VM280 at $280 \mathrm{~cm}^{3} / \mathrm{rev}$. The model error is computed as a normalized summation of squared deviations of modeled and measured friction torques.

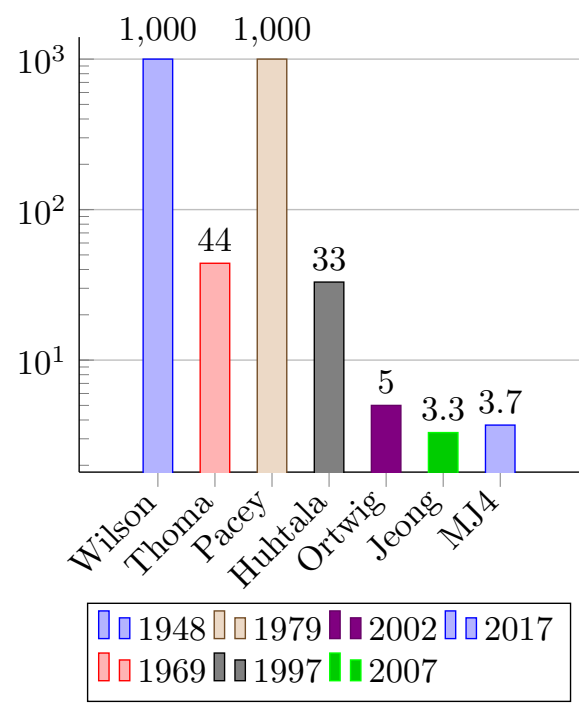

Figure 3: Model error, when optimizing model parameters for fixed displacement. Displacement setting was held constant at maximum value $280 \mathrm{~cm}^{3} / \mathrm{rev}$.

For the fixed motor model identification a complete
$2 \mathrm{D}$ operation area are considered with rotational speed range of $400 \mathrm{rev} / \mathrm{min}$ to $2500 \mathrm{rev} / \mathrm{min}$ and pressures from 50bar to 350bar. The results show that a significant improvement is obtained with Ortwigs and Jeongs model.

Next, the models are investigated for variable displacements. However, not all of the models listed in table 1 are adaptable to variable displacement motors. For example, Wilson and Jeong do not include any variables related to displacement setting. Pacey's model is an extension of Wilson's model so that variable displacement can be handled and, similarly, the modified Jeong models (MJ1...6) presented earlier are extensions of Jeong's model. The displayed error in figure 4 and 5 is an accumulated normalized error for the complete 3D operational range (displacement, pressure drop, angular speed).

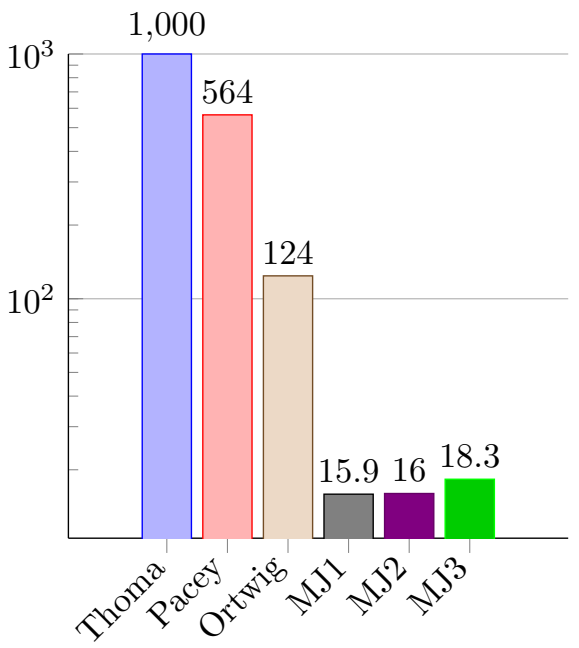

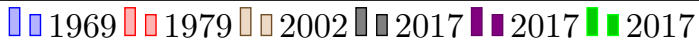

Figure 4: Model error, when optimizing model parameters for variable displacement. Motor type is A6VM 71series size 280.

As can be seen in fig. 4 the new models give a significant better results for this variable axial piston motor. Comparing the different new models (see figure 5), it can be seen that models MJ4, MJ5 and MJ6 are the ones with the best accuracy. 


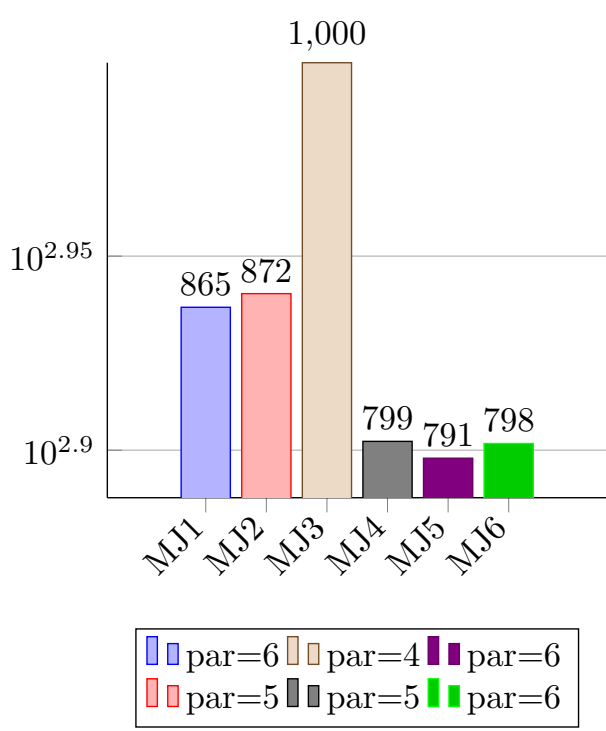

Figure 5: Model error, when optimizing model parameters for variable displacement.

MJ5 has slightly better accuracy but does also have the most parameters (6). MJ4 is seen as the model with the simplest and preferred model structure. This model is used for further investigation of actual model error.

The contributions from the terms $\left(T_{f 1-6}\right)$ in MJ4, MJ5, and MJ6 are shown in figure 6.

$$
\begin{aligned}
T_{f 1} & =C_{g} \cdot \mu \cdot\left|\omega_{m}\right| \\
T_{f 2} & =C_{i} \cdot \rho \cdot\left|\omega_{m}\right|^{C_{n}} \cdot D^{C_{n}+2} \\
T_{f 3} & =C_{f} \cdot \Delta p_{m} \cdot D_{m} \\
T_{f 4} & =C_{e} \\
T_{f 5} & =C_{m} \cdot \Delta p_{m}^{2} \\
T_{f 6} & =C_{k} \cdot \frac{D_{m} \cdot \Delta p_{m}}{D_{m, \max } \cdot\left|\omega_{m}\right|} \cdot \sqrt{\frac{\Delta p_{m}}{\rho}} \\
T_{f}= & T_{f 1}+T_{f 2}+T_{f 3}+T_{f 4}+T_{f 5}+T_{f 6}
\end{aligned}
$$

The contributions are summarized over a range in angular velocity from $n_{1}=300 \mathrm{rev} / \mathrm{min}$ to $n_{2}=$ $2500 \mathrm{rev} / \mathrm{min}$ with steps of $10 \mathrm{rev} / \mathrm{min}$, and then divided by sum of total friction (see eq.20). The two most significant contributors (see fig. 6) in all these models (MJ4, MJ5, MJ6) are the turbulent pressure loss $\left(T_{f 2}\right)$ and the Coulomb friction loss $\left(T_{f 4}\right)$.

$$
T_{f i \%}=\frac{\sum_{n_{1}}^{n_{2}} T_{f i}}{\sum_{i=1}^{i=6} \sum_{n_{1}}^{n_{2}} T_{f i}}
$$

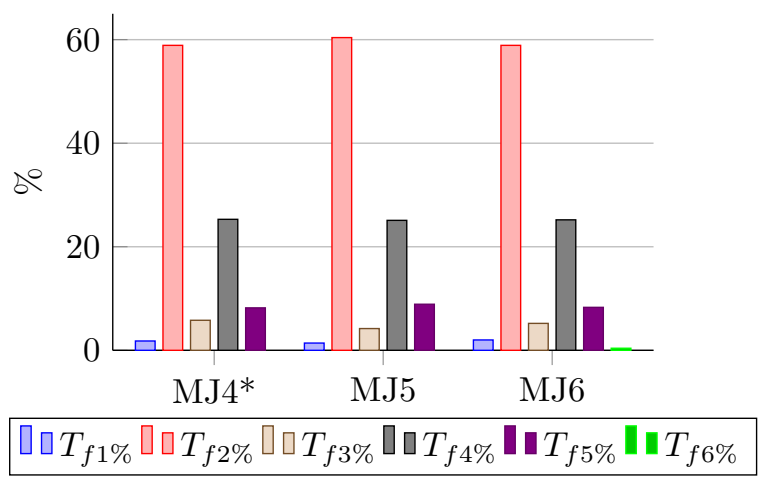

Figure 6: Each term from the friction models in percentage of the accumulated friction force at $200 \mathrm{bar}, 250 \mathrm{~cm}^{3} / \mathrm{rev}$ and angular velocity from 300 to $2500 \mathrm{rev} / \mathrm{min}$.

*For MJ4, $C_{n}$ in $T_{f 2}$ equal 2.

The contribution of these losses are in figure 7 shown in more detail for model MJ4.

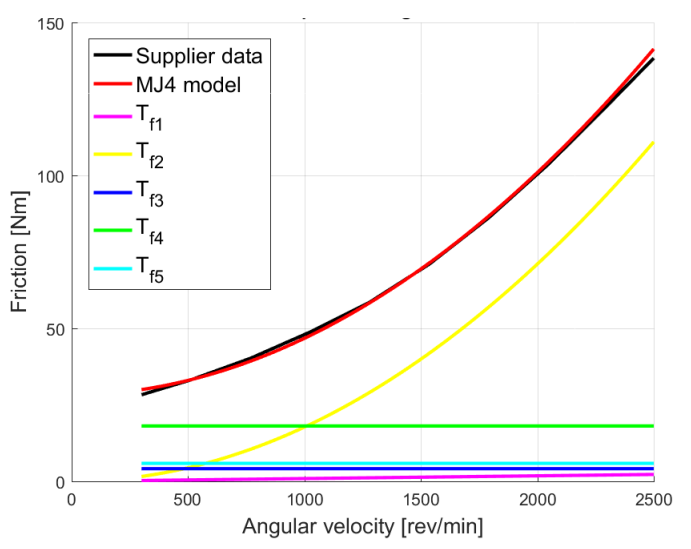

Figure 7: Contributions of the different terms within the motor friction formula MJ4. Motor displacement is set to $250 \mathrm{~cm}^{3} / \mathrm{rev}$ and pressure drop across motor is held constant at 200bar. $C_{n}$ in $T_{f 2}$ equal 2

\section{Results}

The results from figure 8 and 9 show a trend that the friction model error is within $1 \%$ for displacement settings above $150 \mathrm{~cm}^{3} / \mathrm{rev}$ and speeds up to $2500 \mathrm{rev} / \mathrm{min}$. For lower speeds up to $1500 \mathrm{rev} / \mathrm{min}$ the error is typically less than $0.5 \%$. The error is calculated as a percentage of the nominal torque, $T_{N}$.

$$
T_{N}=D_{m} \cdot \Delta p_{m}
$$

The lower displacement settings in figure 9, show that the model becomes less accurate as the displace- 


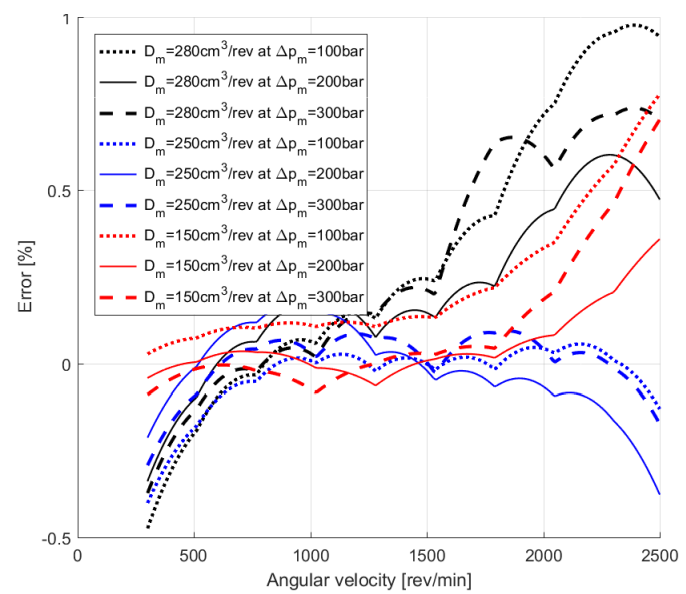

Figure 8: Error between model, MJ4, and the performance data from supplier. Error is measured as a percentage of the nominal motor torque. The comparison is performed at $\Delta p_{m}=100$ bar $\Delta p_{m}=200$ bar and $\Delta p_{m}=$ $300 b a r$.

ment is reduced below $150 \mathrm{~cm}^{3} / \mathrm{rev}$ and also at speeds above $2000 \mathrm{rev} / \mathrm{min}$.

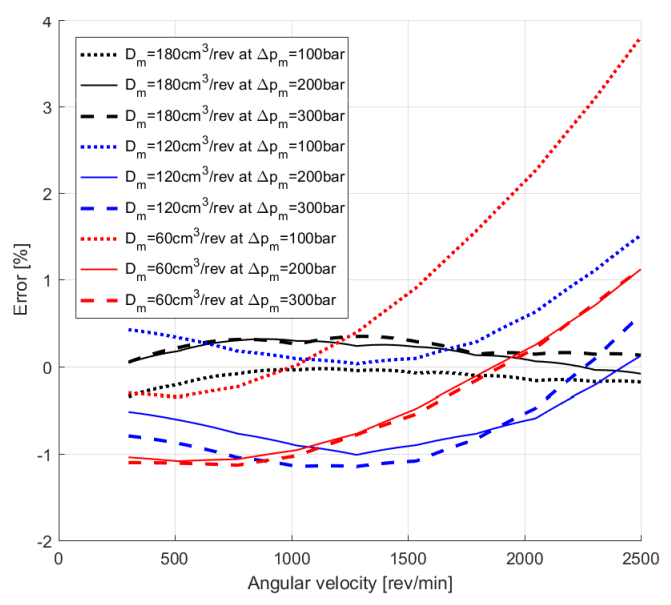

Figure 9: Error between model for low displacement settings, MJ4, and the performance data from supplier. Error is measured as a percentage of the nominal motor torque. The comparison is performed at $\Delta p_{m}=100 \mathrm{bar}$ $\Delta p_{m}=200 \mathrm{bar}$ and $\Delta p_{m}=300 \mathrm{bar}$.

\section{Conclusions}

For simulation purposes the MJ4 model is showed to have a decent accuracy (within 1\% of applied torque) in the range from 400 to $2500 \mathrm{rev} / \mathrm{min}$ and 100 to 300bar. What the model shows is drastically reduced accuracy at motor displacements lower than approx $120 \mathrm{~cm}^{3} /$ rev.

The model tends to have higher accuracy at low speeds near 400rev/min. For speeds lower than 400rev/min the suppliers data-table does not give any information. Stiction forces and friction with mixed lubrication is not represented, but there is reason to believe that it should be detectable at low speeds.

Any temperature dependency is not taken into account and oil density and viscosity is kept constant. The viscosity could be implemented in the formulas, but due to the limited amount of data from supplier it would not be possible to verify their influence.

The model investigation also shows a need for customized models. The MJ4 is good for the Bosch Rextroth 71 series, but fitting the same model to a 63 series $\left(28-200 \mathrm{~cm}^{3} / \mathrm{rev}\right)$ does not achieve the same accuracy. An example is shown in figure 10, where the MJ4, MJ5, and MJ6 and tried fitted to a A6VM S63 $200 \mathrm{~cm}^{3} / \mathrm{rev}$, and a A6VM S71 $215 \mathrm{~cm}^{3} / \mathrm{rev}$.

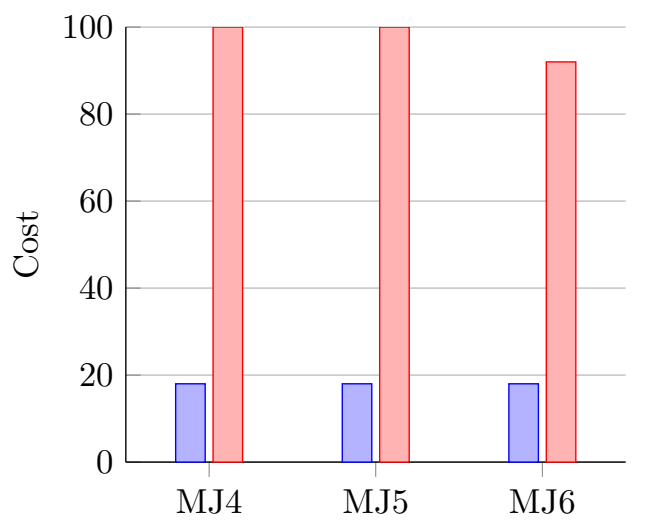

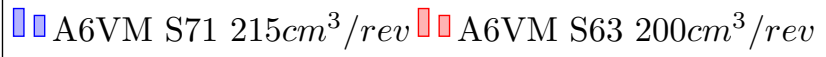

Figure 10: Comparison of two different motors with the same friction model structure. "Cost" is data from the optimization algorithm and is representing the summarized squared error between the supplier data and the model. The data is then normalized from 0 to 100 .

As seen in figure 10 the accuracy differs a lot when comparing it to data from a similar but different hydraulic motor. The difference highlights the complexity of the motors and implies that a general friction model cannot fit all range of hydraulic motors without including more model terms. 


\section{Further work}

The low speed friction has a high influence on driving performance and should be investigated further. Figure 11 shows the cyclic behavior for a winch in heave compensation mode, and as can be seen the winch is passing through the low speed area two times for every wave cycle.

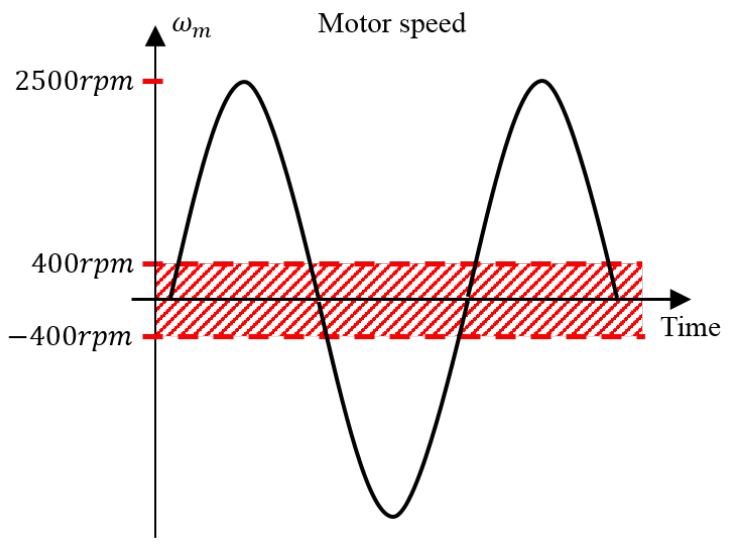

Figure 11: Typical winch speed situation for a winch doing heave compensation.

Since the purpose of this model is closed loop hydraulic crane systems, the current work must be continued with measurement performed on actual cranes with the proposed MJ4 as initial model but with extra terms handling both the remaining friction of the crane power transmission as well as increased motor friction at low-to-zero speed.

\section{References}

Bosch Rexroth AG. Sales Information, Axial Piston Units. 2010.

Dorey, R. Modelling of losses in pumps and mo- tors. First Bath International Fluid Workshop, 1988. URL www. scopus. com.

Huhtala, K. and Villenius, M. Comparison of SteadyState Models of Hydraulic Pump. 1997.

Jeong, H.-S. A novel performance model given by the physical dimensions of hydraulic axial piston motors: model derivation. Journal of Mechanical Science and Technology, 2007. 21(1):83-97. doi:10.1007/BF03161714.

Jeong, H. S. and Kim, H. E. A novel performance model given by the physical dimensions of hydraulic axial piston motors: Experimental analysis. Journal of Mechanical Science and Technology, 2007. 21(4):630-641. doi:10.1007/BF03026968.

Ortwig, H. New Method of Numerical Calculation of Losses and Efficiencies in Hydrostatic. SAE International Off-Highway Congress, 2002. (724).

Pacey, D. A., Turnquist, R. O., and Clark, S. J. The development of a coefficient model for hydrostatic transmissions. In In: Proc.35th Nat.conf.on Fluid Power, volume 33. 35th National Conference in Fluid Power, Chicago, pages 173-178, 1979. URL www. scopus. com.

Schlösser, W. Mathematical model for displacement pump and motors part 2. In Hydraulic power transmission, pages 324-328. London, 1961.

Thoma, J. Mathematical models and effective performance of hydrostatic machines and transmission. Hydraulic and Pneumatic Power, 1969. pages 642651. URL www.scopus.com.

Wilson, W. Performance criteria for positive displacement pumps and fluid motors. In ASME Semiannual Meeting, paper No. 48-SA-14. 1948. 